\title{
The steady flow of one uniformly rotating fluid layer above another immiscible uniformly rotating fluid layer.
}

\author{
P. D. Weidman \\ Department of Mechanical Engineering \\ University of Colorado \\ Boulder, CO 80309-0427 USA \\ M. R. Turner \\ Department of Mathematics \\ University of Surrey \\ Guildford, Surrey GU2 7XH \\ United Kingdom
}

\begin{abstract}
The steady laminar flow of two immiscible, uniformly rotating fluid layers is studied and exact similarity solutions of the axisymmetric Navier-Stokes equations in cylindrical polar coordinates are found. The similarity solutions occur with a flat interface at $z=0$ under the parameter restriction that $\sigma^{2} \rho=1$ where $\sigma$ is the ratio of the fluid angular velocities at $z= \pm \infty$ and $\rho$ is the density ratio of the two fluids. Under this restriction the problem reduces to one with two independent parameters, $\sigma$ and $\mu$, which is the viscosity ratio of the fluids. Numerical results of the resulting system of ODEs are found for selected values of $\mu$ and $\sigma$, and it is shown that similarity solutions exist for $\sigma_{c}(\mu) \leq \sigma \leq 1$ where $\sigma_{c}(\mu)<0$ (i.e. counter-rotating flows). For $\sigma<0$ the lower fluid can become divided into two distinct recirculation regions between which fluid cannot transfer.
\end{abstract}

\section{Introduction}

The investigation of rotating flows has a long history due to their significance in industrial applications, as well as being of fundamental interest. Such fluid flow systems can be wholly confined between two disks, or semi-confined, with the disk/s rotating at a constant angular velocity, and/or with the fluid in the far field undergoing constant rigid body rotation. The study of these axisymmetric flows is motivated by their connection to both ocean circulation models and turbomachinary applications (see e.g Owen and Rogers (1989) and references therein for a review). Further, investigation of these flows is also motivated by theoretical interest, e.g. determining exact solutions to the Navier-Stokes equations in particular geometries and setups.

The theoretical interest in these flows stems from the fact that for laminar flows, axisymmetric, steady, exact solutions to the Navier-Stokes equations can be found in the form of similarity 
solutions. These similarity solutions are of great interest because their existence allows for detailed theoretical studies of the flows, including determining the stability properties of such flows. However, the study of similarity solutions is only significant if they are physically realizable in experiments (Durlofsky and Brady, 1983). For laminar, axisymmetric, steady flows similar to those studied here, there is both numerical and experimental evidence which shows that similarity solutions act as an attractor for flows with weakly unsteady perturbations. For the flow produced by a steadily rotating infinite disk below a stationary fluid, the experiments of Lingwood (1996) confirmed the realization of the similarity solution of von Kármán (1921) to high level of accuracy. The similarity solution of Bödewadt (1940) generated by a rigidly rotating fluid above a fixed infinite disk, is more difficult to realize experimentally due to the difficulty of producing a rigidly rotating infinite flow far from the disk. During their studies investigating flows with finite and infinite disks plus rigid rotation from the disk, Bodonyi and Stewartson (1975) and Stewartson et al. (1982) concluded that the Bödewadt solution exists. Subsequently, by considering the spin-down of a cylindrical cavity, Savaş $(1983,1987)$ showed that the Bödewadt solution is realizable locally at the end walls of the cylinder. This was later confirmed in the numerical calculations and experiments of Lopez and Weidman (1996) and Lopez (1996), the latter study of which considered the spin-down of a cylindrical cavity in which only one end wall was impulsively stopped. It is claimed that this approach 'may be as close as possible to it (Bödewadt flow) in a physically realizable finite domain'.

The flow examples documented above are ones consisting of only one type of fluid. For the case of flows consisting of two immiscible fluid layers, the literature is more limited, with only three problems known to have been investigated for the planar two-dimensional geometry, in which exact similarity solutions exist. The first is by Lock (1951) who considered the velocity distribution between two parallel streams with the same configuration for the Kelvin-Helmholtz instability and calculated similarity solutions for a selection of parameters. The second study by Wang (1992) considered a linear shear flow over a quiescent lower fluid far from the interface. In the third, more recent study, Weidman and Wang (2018) studied the boundary layer that forms at the interface of two different linear shear flows, and an interesting feature discovered of dual similarity solutions for some parameter range. However, only one branch of solutions turned out to be physically realizable.

In the current paper we analyze what may be considered the axisymmetric analogue of Lock's problem, namely the flow generated by one viscous fluid layer (denoted by subscript 1) rotating at constant angular velocity $\omega_{1}$ above a second immiscible viscous fluid layer (denoted by subscript 2 ) rotating at constant angular velocity $\omega_{2}$. This problem is threedimensional and it is found that exact similarity solutions exist in the restricted case $\rho \sigma^{2}=1$, 
where $\rho=\rho_{2} / \rho_{1}$ is the density ratio of the two fluids and $\sigma=\omega_{2} / \omega_{1}$. Here $\sigma>0$ means the flows are co-rotating while $\sigma<0$ denotes counter-rotating flows. We note at the outset that this problem has not been considered in the books on two-fluid flows by Joseph and Renardy (1992).

The presentation of the paper is as follows. The problem is formulated in $\S 2$ and numerical results for various values of $\mu=\mu_{2} / \mu_{1}$, the viscosity ratio of the two fluids, and $\sigma$ are presented in $\S 3$, along with velocity profiles in the radial, azimuthal and axial directions. The paper concludes with a discussion in $\S 4$.

\section{Formulation}

Consider an incompressible upper fluid with velocity $\mathbf{u}_{1}=\left(u_{1}, v_{1}, w_{1}\right)$, pressure $p_{1}$, density $\rho_{1}$ and viscosity $\mu_{1}$ rotating at constant angular velocity $\omega_{1}$ above an immiscible, incompressible lower fluid with velocity $\mathbf{u}_{2}=\left(u_{2}, v_{2}, w_{2}\right)$, pressure $p_{2}$, density $\rho_{2}$ and viscosity $\mu_{2}$ rotating at constant angular velocity $\omega_{2}$. The velocity components are with respect to a cylindrical coordinate system $(r, \theta, z)$ and gravity is assumed to act parallel to the $z$-axis. See figure 1 for a schematic of the system considered. Note that solutions are assumed to be steady and axisymmetric and that the interface is assumed to be flat, which means the effect of surface tension on the results can be neglected as there is zero interface curvature.

Non-dimensionalizing lengths with $\sqrt{\nu_{1} / \omega_{1}}$, velocities with $\sqrt{\nu_{1} \omega_{1}}$, and pressure with $\rho_{1} \nu_{1} \omega_{1}$, where $\nu_{1}=\mu_{1} / \rho_{1}$ is the kinematic viscosity in the upper layer, one arrives at the dimensionless Navier-Stokes equations in the upper layer as

$$
\begin{gathered}
\left(\mathbf{u}_{1} \cdot \nabla\right) \mathbf{u}_{1}=-\nabla\left(p_{1}+F^{-1} \eta\right)+\nabla^{2} \mathbf{u}_{1} \quad(\eta \geq 0) \\
\nabla \cdot \mathbf{u}_{1}=0
\end{gathered}
$$

and in the lower layer one finds

$$
\begin{gathered}
\left(\mathbf{u}_{2} \cdot \nabla\right) \mathbf{u}_{2}=-\frac{1}{\rho} \nabla\left(p_{2}+\rho F^{-1} \eta\right)+\frac{\mu}{\rho} \nabla^{2} \mathbf{u}_{2} \quad(\eta \leq 0) \\
\boldsymbol{\nabla} \cdot \mathbf{u}_{2}=0 .
\end{gathered}
$$

Here the dimensionless axial variable is $\eta=z / \sqrt{\nu_{1} / \omega_{1}}, F=\left(\omega_{1} \sqrt{\nu_{1} \omega_{1}}\right) / g$ is the Froude number and in $(1 c)$ there appears the density and viscosity ratios

$$
\rho=\frac{\rho_{2}}{\rho_{1}}, \quad \mu=\frac{\mu_{2}}{\mu_{1}} .
$$

We posit the following similarity form for the upper fluid layer satisfying continuity

$$
u_{1}(r, \eta)=r f_{1}^{\prime}(\eta), \quad v_{1}(r, \eta)=r g_{1}(\eta), \quad w_{1}(r, \eta)=-2 f_{1}(\eta)
$$


and that for the lower fluid layer is

$$
u_{2}(r, \eta)=r f_{2}^{\prime}(\eta), \quad v_{2}(r, \eta)=r g_{2}(\eta), \quad w_{2}(r, \eta)=-2 f_{2}(\eta)
$$

Application of far-field boundary conditions of constant rotation at $\omega_{1}$ in the upper layer and constant rotation at $\omega_{2}$ in the lower layer gives the far-field conditions in the upper layer

$$
f_{1}^{\prime}(\infty)=0, \quad g_{1}(\infty)=1
$$

and in the lower layer

$$
f_{2}^{\prime}(-\infty)=0, \quad g_{2}(-\infty)=\sigma
$$

where the new dimensionless quantity is the ratio

$$
\sigma=\frac{\omega_{2}}{\omega_{1}}
$$

Inserting the similarity forms (2) into the Navier-Stokes equations (1) leads to a pair of coupled ODEs in the upper layer

$$
\begin{gathered}
f_{1}^{\prime \prime \prime}+2 f_{1} f_{1}^{\prime \prime}-f_{1}^{\prime 2}+g_{1}^{2}-1=0 \\
g_{1}^{\prime \prime}+2\left(f_{1} g_{1}^{\prime}-f_{1}^{\prime} g_{1}\right)=0
\end{gathered}
$$

with pressure field obtained from integrating the pair of equations (1a) as

$$
p_{1}(r, \eta)=p_{10}+\frac{r^{2}}{2}-2\left[f_{1}^{2}(\eta)+f_{1}^{\prime}(\eta)\right]-F^{-1} \eta
$$

where $p_{10}=p_{1}(0,0)$ is a constant. In the lower layer the coupled ODE system is

$$
\begin{gathered}
\frac{\mu}{\rho} f_{2}^{\prime \prime \prime}+2 f_{2} f_{2}^{\prime \prime}-f_{2}^{\prime 2}+g_{2}^{2}-\sigma^{2}=0 \\
\frac{\mu}{\rho} g_{2}^{\prime \prime}+2\left(f_{2} g_{2}^{\prime}-f_{2}^{\prime} g_{2}\right)=0
\end{gathered}
$$

with pressure field obtained from integrating equations (1c) as

$$
p_{2}(r, \eta)=p_{20}+\rho\left[\sigma^{2} \frac{r^{2}}{2}-2\left(f_{2}^{2}(\eta)+\frac{\mu}{\rho} f_{2}^{\prime}(\eta)\right)\right]-\rho F^{-1} \eta
$$

where again $p_{20}=p_{2}(0,0)$ is a constant.

The kinematic interfacial conditions stipulating continuity of the velocity field across the flat interface give

$$
f_{1}(0)=f_{2}(0)=0, \quad f_{1}^{\prime}(0)=f_{2}^{\prime}(0), \quad g_{1}(0)=g_{2}(0)
$$


Then application of the interface stress jump conditions

$$
\left[\tau_{z r}\right]=0, \quad\left[\tau_{z \theta}\right]=0, \quad\left[\tau_{z z}\right]=0
$$

give the following results

$$
\begin{aligned}
& g_{1}^{\prime}(0)=\mu g_{2}^{\prime}(0) \\
& f_{1}^{\prime \prime}(0)=\mu f_{2}^{\prime \prime}(0)
\end{aligned}
$$

and

$$
p_{2}(r, 0)-p_{1}(r, 0)=4\left[f_{1}^{\prime}(0)-\mu f_{2}^{\prime}(0)\right]
$$

The systems of ODEs in each layer, (4) and (5), comprise of two $5^{\text {th }}$ order systems, and hence require ten boundary and interfacial conditions for solution. In (3), (6) and (7) we have eleven conditions so the system appears over specified; however inserting the expressions for the pressures from $(4 \mathrm{c})$ and $(5 \mathrm{c})$ into $(7 \mathrm{c})$ and applying $f_{1}(0)=f_{2}(0)=0$ gives, after simplification, the result

$$
p_{20}-p_{10}+\frac{r^{2}}{2}\left(\rho \sigma^{2}-1\right)+2\left[\mu f_{2}^{\prime}(0)-f_{1}^{\prime}(0)\right]=0
$$

We now apply $f_{1}^{\prime}(0)=f_{2}^{\prime}(0)$ from (6) and set coefficients of powers of $r$ to zero to obtain

$$
p_{20}-p_{10}=2(1-\mu) f_{1}^{\prime}(0)
$$

and the critical relation

$$
\rho \sigma^{2}=1
$$

which is required for self-similarity. Thus (7c) is automatically satisfied if (9) holds and hence for this restriction of the parameter $\sigma$ we have a well defined problem.

Reiterating the coupled system of equations, for $\eta>0$ we have

$$
\begin{gathered}
f_{1}^{\prime \prime \prime}+2 f_{1} f_{1}^{\prime \prime}-f_{1}^{\prime 2}+g_{1}^{2}-1=0 \\
g_{1}^{\prime \prime}+2\left(f_{1} g_{1}^{\prime}-f_{1}^{\prime} g_{1}\right)=0
\end{gathered}
$$

while for $\eta<0$ we have

$$
\begin{gathered}
\mu \sigma^{2} f_{2}^{\prime \prime \prime}+2 f_{2} f_{2}^{\prime \prime}-f_{2}^{\prime 2}+g_{2}^{2}-\sigma^{2}=0 \\
\mu \sigma^{2} g_{2}^{\prime \prime}+2\left(f_{2} g_{2}^{\prime}-f_{2}^{\prime} g_{2}\right)=0
\end{gathered}
$$

where $\rho=1 / \sigma^{2}$ has been eliminated. These are to be solved with far-field conditions

$$
\begin{gathered}
f_{1}^{\prime}(\infty)=0, g_{1}(\infty)=1 \\
f_{2}^{\prime}(-\infty)=0, g_{2}(-\infty)=\sigma
\end{gathered}
$$


along with the interfacial conditions

$$
\begin{gathered}
f_{1}(0)=f_{2}(0)=0, f_{1}^{\prime}(0)=f_{2}^{\prime}(0), g_{1}(0)=g_{2}(0) \\
\mu f_{2}^{\prime \prime}(0)=f_{1}^{\prime \prime}(0), \mu g_{2}^{\prime}(0)=g_{1}^{\prime}(0) .
\end{gathered}
$$

Thus the steady solution of two fluids in solid body rotation is governed by two parameters: $\mu>0$ and $\sigma$. For stability without rotation in either layer in the presence of gravity, we take

$\rho \geq 1$ which restricts the angular velocity ratio to $0 \leq \sigma^{2} \leq 1$. Note that the lower layer can counter-rotate relative to the upper layer. The case when $\sigma=0($ or $\rho=\infty)$ corresponds to replacing the lower fluid with a solid wall at $\eta=0$. Hence in the limit $\sigma \rightarrow 0$ we expect flow variables, such as the shear stresses at $\eta=0$, to tend to those of the Bödewadt problem (Bödewadt, 1940).

\section{Results}

In this section we present numerical solutions of the system of equations (10)-(11) with farfield and interfacial conditions (12) and (13), respectively, for various values of $\mu$ and $\sigma$. To aid the numerical computations for small values of $\mu \sigma^{2}$, where the lower layer ODEs become singular, we introduce the following change of variables in layer 2, viz.

$$
\zeta=-\mu^{-1 / 2} \sigma^{-1} \eta, \quad F_{2}(\zeta)=\mu^{-1 / 2} \sigma^{-1} f_{2}(\eta), \quad G_{2}(\zeta)=g_{2}(\eta)
$$

which removes the singular term from highest derivative, and changes the integration range to $\zeta \in[0, \infty]$ (the same as the $\eta$ domain) which means that both sets of equations can now be solved simultaneously on the same interval. The equations are solved by numerically integrating from the interface at $\eta=0$ out to $\eta=\eta_{\max }$ and the four interfacial unknowns $f_{1}^{\prime}(0), f_{1}^{\prime \prime}(0), g_{1}(0), g_{1}^{\prime}(0)$ are updated via Newton iterations using the far-field conditions (12). For the results presented we integrated out to $\eta_{\max }=20$ with a step-size $\Delta \eta=10^{-4}$ in order to obtain converged, grid-independent results.

For small and negative values of $\sigma$, integrating to $\eta_{\max }=20$ becomes more troublesome as the solutions are more sensitive to the accuracy of the interface unknowns. This can cause the shooting approach to fail. To overcome this we use the shooting-splitting method of Firnett and Troesch (1974) which splits the integral domain into $N$ sub-domains. In each sub-domain the unknown variables at the smaller $\eta$ end of the sub-domain are integrated to the junction with the next sub-domain and then these unknowns are updated, via Newton iterations, using the continuity of the solution and its derivatives across sub-intervals. This process continues until the results converge. This approach leads to a larger set of nonlinear equations to solve than the simple shooting approach $(10 N \times 10 N$ in this case $)$ but the 
exponential growth of the solution is now restricted in each sub-domain and hence there is higher chance of convergence. We find $10 \leq N \leq 20$ for this problem is sufficient to gain the converged solutions presented below.

\subsection{Positive $\sigma$ Results}

We have chosen viscosity ratios $\mu=\{0.1,0.2,0.5,1.0,2.0,5.0,10.0\}$ to present the overall behaviour of the flow. First we display the upper layer radial stress parameter $f_{1}^{\prime \prime}(0)$ as a function of $\sigma$ in figure 2 and the variation of the upper layer azimuthal stress parameter $g_{1}^{\prime}(0)$ is shown in figure 3 . These show that as the viscosity of the lower layer is increased $(\mu$ increasing) both the radial and azimuthal stresses at the interface increase in magnitude for fixed $\sigma$. As $\sigma \rightarrow 0$ we observe that all the results agree with the Bödewadt (1940) result

$$
f_{1}^{\prime}(0)=g_{1}(0)=0, \quad f_{1}^{\prime \prime}(0)=-0.94197 \quad g_{1}^{\prime}(0)=0.77289
$$

The radial and azimuthal interfacial velocities $f_{1}^{\prime}(0)$ and $g_{1}(0)$ on the other hand decrease in absolute magnitude at a fixed $\sigma$ for increasing $\mu$, as seen in figures 4 and 5 respectively. The radial interfacial velocity is always directed towards the origin $\left(f_{1}^{\prime}(0)<0\right)$ for all values

of $0 \leq \sigma \leq 1$, and reaches a maximum absolute magnitude somewhere in $0 \leq \sigma \lesssim 0.2$. However, the value of $f_{1}^{\prime}(0)$ for $\sigma>0$ is an order of magnitude smaller than the azimuthal interface velocity $g_{1}(0)$.

In figures 6 and 7 we present the upper layer far-field axial velocity parameter $f_{1}(\infty)$ and the lower layer far-field axial velocity parameter $f_{2}(-\infty)$, respectively. These show that in the upper layer the fluid in the far-field is directed away from the interface $\left(w_{1}>0\right)$ while in the lower layer the fluid is directed towards the interface $\left(w_{2}>0\right)$. Therefore, to conserve mass, the upper layer must have a net inflow of fluid spiraling in from $r=\infty$ close to $\eta=0$, while for the lower layer there must be a corresponding net outflow spiraling out towards $r=\infty$. This inflow/outflow behaviour either side of $\eta=0$ creates a strong shear layer across this interface as seen in $\S 3.3$. Note that in an oceanographical context, the upper layer motion is synonymous with Ekman pumping, with fluid pumped in along the interface and out to $\eta=\infty$, while in the lower motion is synonymous with Ekman suction, with fluid sucked up from $\eta=-\infty$ and out along the interface (Gill, 1982). Comparing this result to two of the most common rotating flow solutions, the von Kármán flow has an inflow of fluid from $\eta=\infty$ while the Bödewadt flow has an outflow of fluid at $\eta=\infty$. Hence there appears to be similarities of the upper layer flow and the Bödewadt result, and the lower layer flow and the von Kármán result. This is further explored in $§ 3.3$. 


\subsection{Negative $\sigma$ Results}

Here again we present results for the same viscosity ratios as in §3.1. The upper layer radial stress parameter $f_{1}^{\prime \prime}(0)$ is plotted in figure 8 as a function of $\sigma$ while the upper layer azimuthal stress parameter $g_{1}^{\prime}(0)$ is plotted in figure 9 . Here, for each value of $\mu$, the absolute magnitudes of $f_{1}^{\prime \prime}(0)$ and $g_{1}^{\prime}(0)$ increase with decreasing $\sigma$, down to some critical value $\sigma=\sigma_{c}$, where the numerical scheme fails to converge. For $\sigma<\sigma_{c}$ there are no similarity solutions of the form (2). The radial and azimuthal interfacial velocities are given in figures 10 and 11 respectively, and both increase in absolute magnitude down to $\sigma=\sigma_{c}$. Here we observe that $g_{1}(0)$ has now switched sign from the $\sigma>0$ case in $\S 3.1$ while all other interfacial parameters retain the same sign.

The upper layer far-field axial velocity parameter $f_{1}(\infty)$ and the lower layer far-field axial velocity parameter $f_{2}(-\infty)$ are presented in figures 12 and 13 . As in $\S 3.1$ for $\sigma>0$, the upper layer far-field flow remains directed away from the interface. The absolute magnitude of this outflow increases initially as $\sigma$ reduces from zero to attain a maximum magnitude (minimum of $f_{1}(\infty)$ ) before decreasing in absolute magnitude as $\sigma \rightarrow \sigma_{c}$. In the lower layer, on the other hand, an outflow $\left(w_{2}<0\right)$ is observed for small $|\sigma|$ at all values of $\mu$. In the results presented, for $\mu \leq 2$ this outflow switches back to an inflow as $\sigma \rightarrow \sigma_{c}$, while for $\mu=10$ the flow remains an outflow as $\sigma \rightarrow \sigma_{c}$. For intermediate cases, such as $\mu=5$, the axial flow at $\eta=-\infty$ can alternate between an outflow, to an inflow and back to an outflow as $\sigma$ decreases from zero. Finally, the variation of the limiting negative values $\sigma_{c}$ plotted as a function of $\mu$ in figure 14 shows that this value decreases as $\mu$ increases.

\subsection{Velocity Profiles}

Here we present the radial and azimuthal velocity profiles for $\mu=1$ at $\sigma=\{-0.1,0.0,0.2,0.5,0.8\}$. In all cases we plot both upper and lower layer results together to show the transition across the $\eta=0$ interface. The radial velocity profiles plotted in figure 15 show that the radially inward 'wall' jet above the interface increases significantly as $\sigma$ is reduced to $\sigma=-0.1$, and there is a weak radially outward 'wall' jet just below the interface for all values of $\sigma$. In figure 16 the azimuthal profiles exhibit an overshoot in the upper layer close to the interface whose magnitude increases with decreasing $\sigma$. Note that all velocities are positive for $\sigma>0$ but that for $\sigma=-0.1$ there is the expected counter rotation exhibited in the lower layer. These overshoots in the azimuthal velocity profile lead to a large shear flow across the interface which ultimately leads to the breakdown of the similarity solution.

As mentioned at the end of $\S 3.1$ there are distinct similarities between the upper layer flow and the Bödewadt flow, and similarities between the lower layer flow and the von 
Kármán flow. These similarities are also evident in figures 15 and 16. Both the radial and azimuthal velocity profiles in the upper layer oscillate about their $\eta \rightarrow \infty$ value, for all $\sigma$ values, in the same way as the Bödewadt solution does (see MacKerrell (2005) for velocity profiles). In the lower layer the radial velocity increases to a maximum value before decaying exponentially to zero, while the azimuthal component decays exponentially (with a small amount of overshoot) to its far-field value, akin to the profiles for the von Kármán flow (see Healey (2006) for velocity profiles).

Finally, in figure 17 we plot profiles for the axial velocity $w=-2 f(\eta)$. One observes that this velocity is positive in both the upper and lower layers except for $\sigma=-0.1$ which has an overshoot to negative values near the interface. This region of negative axial flow below the interface signifies a separate region of recirculating flow in which the fluid cannot escape to $\eta=-\infty$ (due to $-2 f_{2}(\eta)=0$ at $\eta=-0.67$ ). This isolated layer of fluid motion is in contrast to the $\sigma>0$ case where $-2 f_{1}(\eta)=0$ only at $\eta=0$.

\section{Discussion and Conclusion}

We have investigated the stable laminar flow generated by one incompressible fluid layer rotating with constant angular velocity $\omega_{1}$ above a second immiscible, incompressible fluid layer rotating with constant angular velocity $\omega_{2}$. By seeking exact solutions to the axisymmetric Navier-Stokes equations in the form of similarity solutions we find the problem can be formulated as four coupled ODEs, dependent upon two parameters: $\mu=\mu_{2} / \mu_{1}$ and $\sigma=$ $\omega_{2} / \omega_{1}$, where $\mu_{i}$ for $i=1,2$ is the dynamic viscosity in each layer. Matching the velocities and stresses of the flows across the interface at $\eta=0$ we find similarity solutions under the restriction $\sigma^{2} \rho=1$ where $\rho=\rho_{2} / \rho_{1}$ is the density ratio, and $-1 \leq \sigma \leq 1$ for static stability under the influence of gravity.

Numerical results show that the similarity solutions exist for $\sigma_{c}(\mu) \leq \sigma \leq 1$ where $\sigma_{c}(\mu)<$ 0 , i.e. for all co-rotating flows similarity solutions exist, but only weak counter-rotation can support such a solution. Also, for co-rotating flows the far-field axial velocities are always transported in the same direction (away from the interface as $\eta \rightarrow \infty$ and toward the interface as $\eta \rightarrow-\infty$ ), while for counter-rotating flows these axial flows can both transport away from the interface depending on the value of $\sigma$. Another interesting feature for counter-rotating flows is an additional layer of recirculating fluid motion can form below the interface (i.e. $w_{2}=0$ at $\eta=0$ and $\eta=-\eta_{0}$ ), in contrast to co-rotating flows where the axial velocity is only zero at $\eta=0$. This behaviour for $\sigma<0$ is dependent on $\mu$.

An interesting comparison is made here for fluid uniformly rotating at angular velocity $\omega$ above an infinite disk rotating at angular velocity $\Omega$ for which we define $\sigma=\omega \Omega$. Rogers and 
Lance (1960) studied the problem of co-rotation and found solutions over the entire range $0 \leq \sigma<\infty$; thus in the range $\sigma \leq 0 \leq 1$ solutions are found for both the fluid-disk problem and the fluid-fluid problem. For counter-rotating flow, Rogers and Lance found solutions down to $\sigma=-0.15$ but after that encountered difficulties. This was further studied by Weidman and Redekopp (1976) who found that a singularity appears at $\sigma \simeq-0.154$; thus like the fluid-fluid problem, the fluid-disk problem has only a small range of negative values of $\sigma$ for which solutions are possible.

It is noted that the same limited range of similarity solutions has also been observed in the Falkner-Skan boundary layer flow (Drazin and Reid, 2004). This breakdown of the similarity solution for $\sigma<\sigma_{c}$ is interesting and warrants further study. The failure is likely due to the radial structure breaking down due to the large shear at the interface creating steady regions of recirculation and hence non-similarity, or because no solutions with a flat interface $(\eta=h(r, \theta)=0)$ exist, in which case the shape of the interface also needs to be determined as part of the solution. It could also be possible that no steady solutions exist.

Throughout this paper the focus has been on calculating the existence of exact, steady, laminar, similarity solutions to the Navier-Stokes equations. These steady solutions act as an attractor for unsteady solutions and the existence of these laminar solutions in practical experiments depends on the stability properties of the flow. As we observed, the velocity profiles have a curious form, combining features of both the von Kármán and Bödewadt flows below and above the interface respectively. From this observation alone, we would expect this flow to have stability properties related to these two flows, i.e. we would expect them to contain both convective and absolute instability features which have been widely documented in the literature on these flows (see Savaş (1983,1987); Pikhtov and Smirnov (1992); Lopez and Weidman (1996); Lingwood (1997); Serre et al (2001); Schouveiler et al (2001) and references therein). In the stability calculation scenario, waves are assumed to have formed on the fluid interface, thus flow features such as surface tension become significant in determining whether the laminar flow is physically realizable or whether the flow breaks up into turbulence. The study of the linear stability of these flows is left as future work so as not to detract attention from the significant new exact solutions to the axisymmetric Navier-Stokes equations presented here. 


\section{References}

Bödewadt, U. T., Die Drehströmung “über festem Grunde, Z. Angew Math. Mech., 20, 241-253 (1940).

Bodonyi, R. J. and Stewartson, K., Boundary-layer similarity near the edge of a rotating disk, J. Appl. Mech., 42(3), 584-90 (1975).

Drazin, P. G. and Reid, W. H., Hydrodynamic stability, (Cambridge university press, Cambridge, 2004).

Durlofsky, L. and Brady, J. F., The spatial stability of a class of similarity solutions, Phys. Fluids, 27(5), 1068-1076 (1984).

Firnett, P. J. and Troesch, B. A., Shooting-splitting method for sensitive two-point boundary value problems, In: Bettis D.G. (eds) Proceedings of the Conference on the Numerical Solution of Ordinary Differential Equations, 362, 408-433 (Springer-Verlag, Berlin, 1974).

Gill, A. E., Atmosphere-ocean dynamics, (Academic, San Diego, 1982).

Healey, J. J., Inviscid long-wave theory for the absolute instability of the rotating-disc boundary layer, Phil. Trans. R. Soc. A, 462(2069), 1467-1492 (2006).

Joseph, D. D. and Renardy, Y., Fundamentals of Two-Fluid Dynamics, Parts I and II, Interdisciplinary Applied Math 4, (Springer-Verlag, New York, 1992).

Kármán, Th. von, Über laminare und turbulente Reibung, Z. Angew Math. Mech., 1, 233-252 (1921).

Lingwood, R. J., An experimental study of absolute instability of the rotating-disk boundarylayer flow, J. fluid Mech., 314, 373-405 (1996).

Lingwood, R. J., Absolute instability of the Ekman layer and related rotating flows, J. fluid Mech., 331, 405-428 (1997).

Lock, R. C., The velocity distribution in the laminar boundary layer between parallel streams, Q. J. Mech. Math., 4, 42-61 (1951).

Lopez, J. M., Flow between a stationary and a rotating disk shrouded by a co-rotating cylinder, Phys. Fluids, 8(10), 2605-2613 (1996).

Lopez, J. M. and Weidman, P. D., Stability of stationary endwall boundary layers during spin-down, J. Fluid Mech., 326, 373-398 (1996). 
MacKerrell, S. O., Stability of Bödewadt flow, Phil. Trans. R. Soc. A, 363(1830), 1181-1187 (2005).

Owen, J. M. and Rogers, R. H., Flow and heat transfer in rotating-disc systems (Wiley, New York, 1998).

Pikhtov, S. V. and Smirnov, E. M., Boundary layer stability on a rotating disk with corotation of the surrounding fluid, Fluid Dyn., 27(5), 657-663 (1992).

Savaş, Ö., Circular waves on stationary disk in rotating flow, Phys. Fluids, 26, 3445-3448 (1983).

Savaş, Ö., Stability of Bödewadt flow, J. Fluid Mech., 183, 77-94 (1987).

Schouveiler, L., Le Gal, P. and Chauve, M. P., Instabilities of the flow between a rotating and a stationary disk, J. Fluid Mech.,443, 329-350 (2001).

Serre, E., Crespo Del Arco, E. and Bontoux, P., Annular and spiral patterns in flows between rotating and stationary discs, J. Fluid Mech., 434, 65-100 (2001).

Stewartson, K., Simpson, C. J. and Bodonyi, R. J., The unsteady boundary layer on a rotating disk in a counter-rotating fluid. Part 2, J. Fluid Mech., 121, 507-515 (1982).

Wang, C. Y., The boundary layers due to shear flow over a still fluid, Phys. Fluids A: Fluid Dynamics, 4, 1304-1306 (1992).

Weidman, P. D. and Redekopp, L. G., On the motion of a rotating fluid in the presence of an infinite rotating disk, Arch. Mech., 24, 1011-1024 (1976).

Weidman, P. D. and Wang, C. Y., Boundary layers at the interface of two different shear flows, Phys. Fluids, 30, 053604 (2018). 


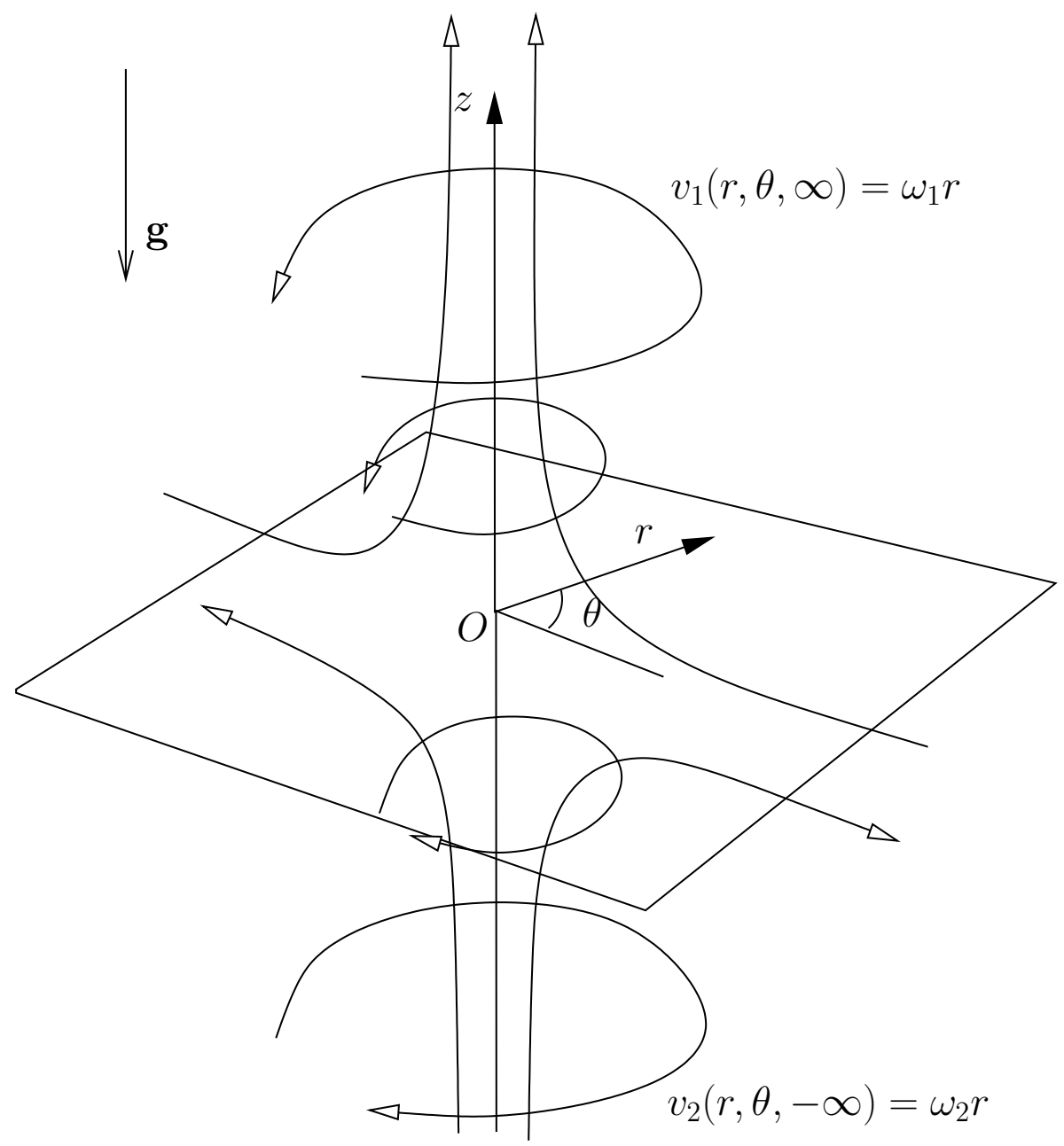

Figure 1. Schematic diagram of the two-fluid rotating flow system considered. This figure depicts a counter-rotating case. 


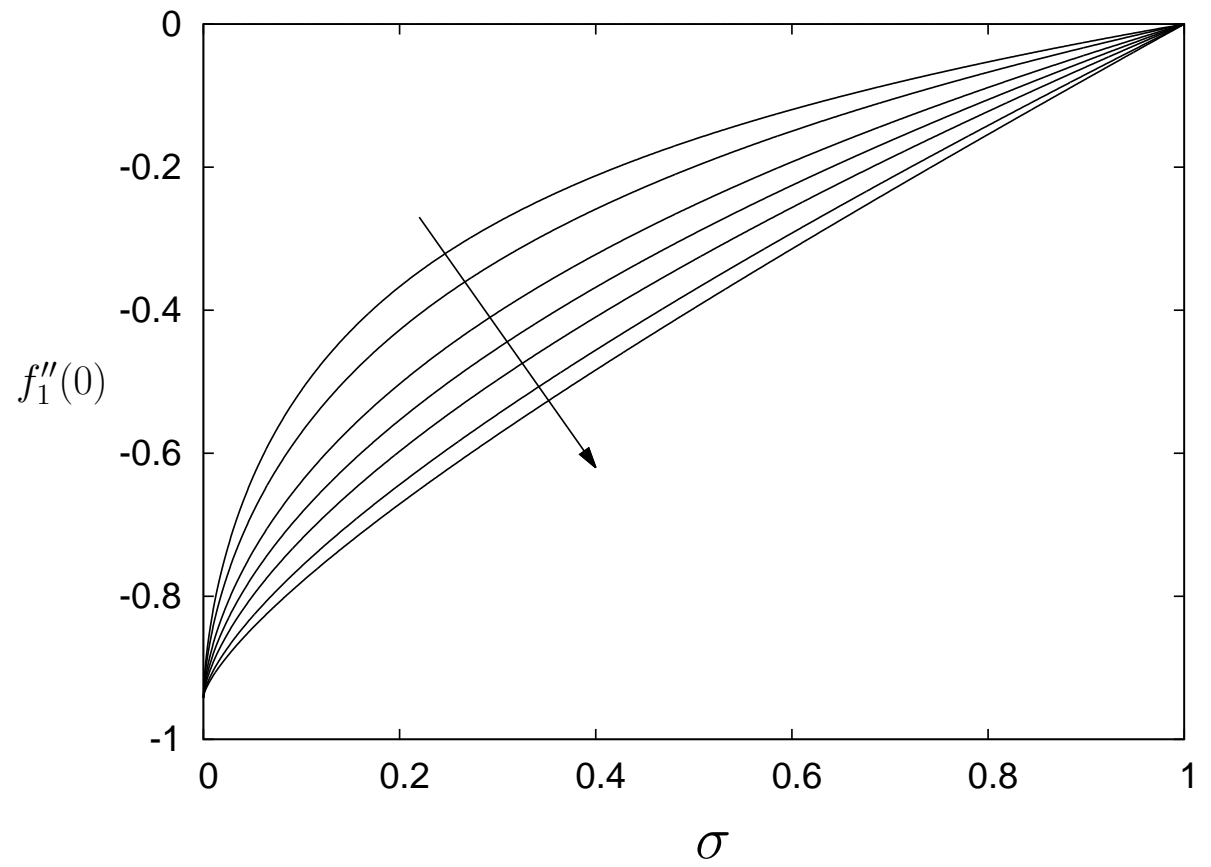

Figure 2. Variation of upper interfacial radial stress parameter $f_{1}^{\prime \prime}(0)$ with positive values of $\sigma$ at $\mu=\{0.1,0.2,0.5,1.0,2.0,5.0,10.0\}$ with the arrow in the direction of increasing values of $\mu$.

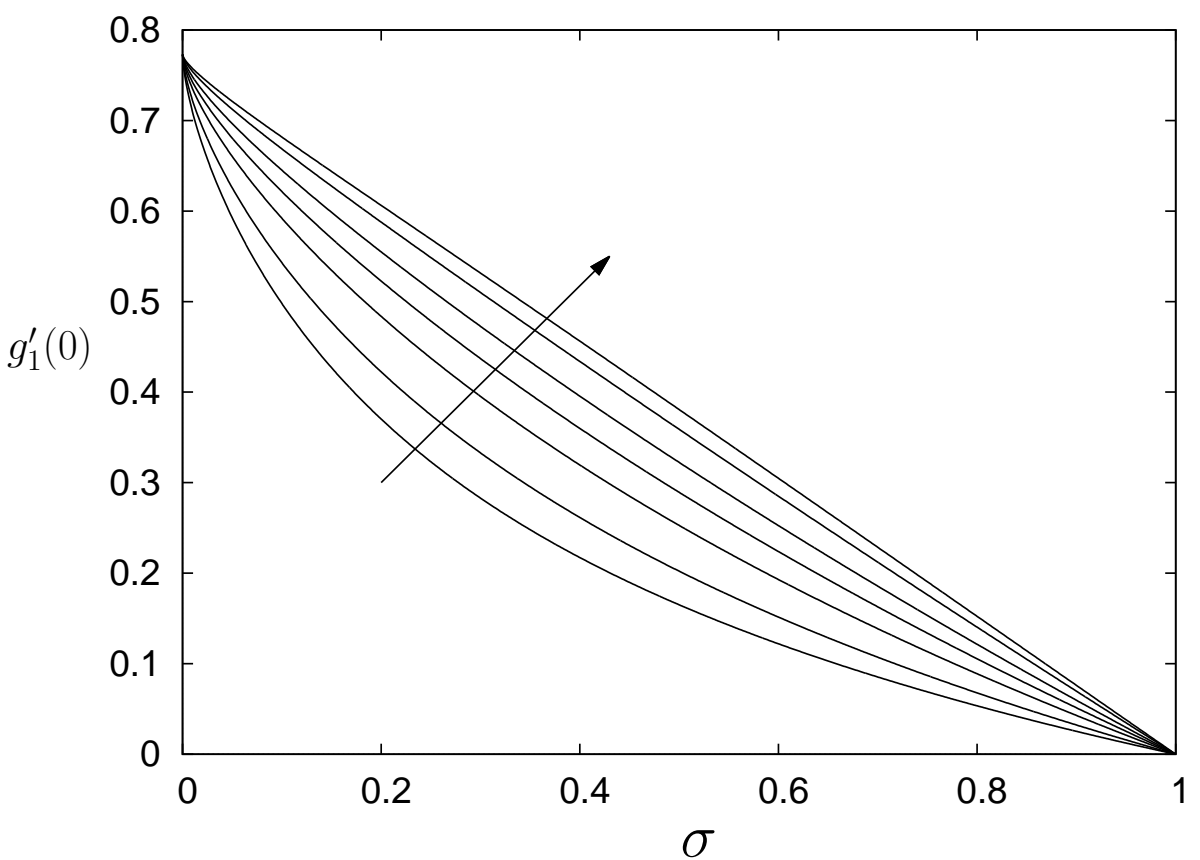

Figure 3. Variation of upper interfacial azimuthal stress parameter $g_{1}^{\prime}(0)$ with positive values of $\sigma$ at $\mu=\{0.1,0.2,0.5,1.0,2.0,5.0,10.0\}$ with the arrow in the direction of increasing values of $\mu$. 


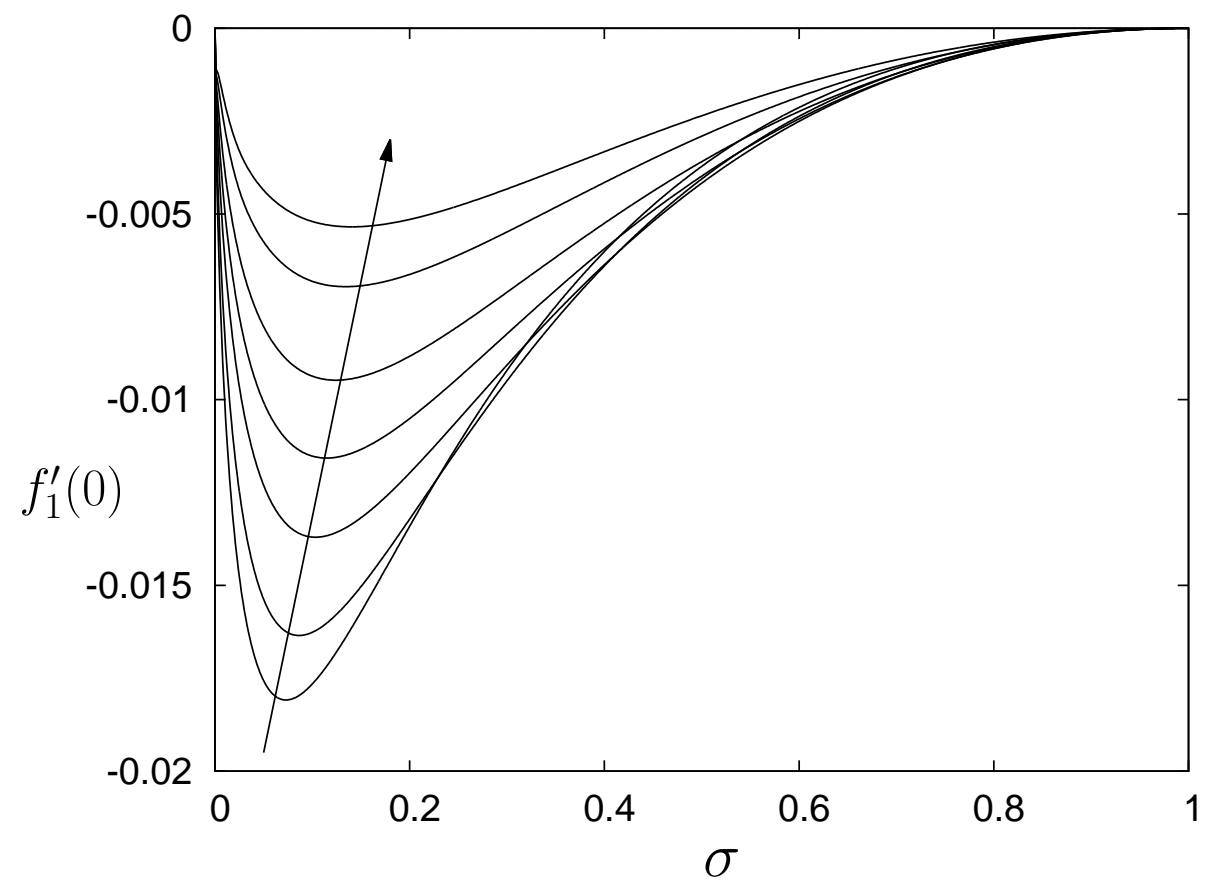

Figure 4. Upper layer interfacial radial velocity $f_{1}^{\prime}(0)$ for positive values of $\sigma$ at $\mu=\{0.1,0.2,0.5,1.0,2.0,5.0,10.0\}$ with the arrow in the direction of increasing values of $\mu$.

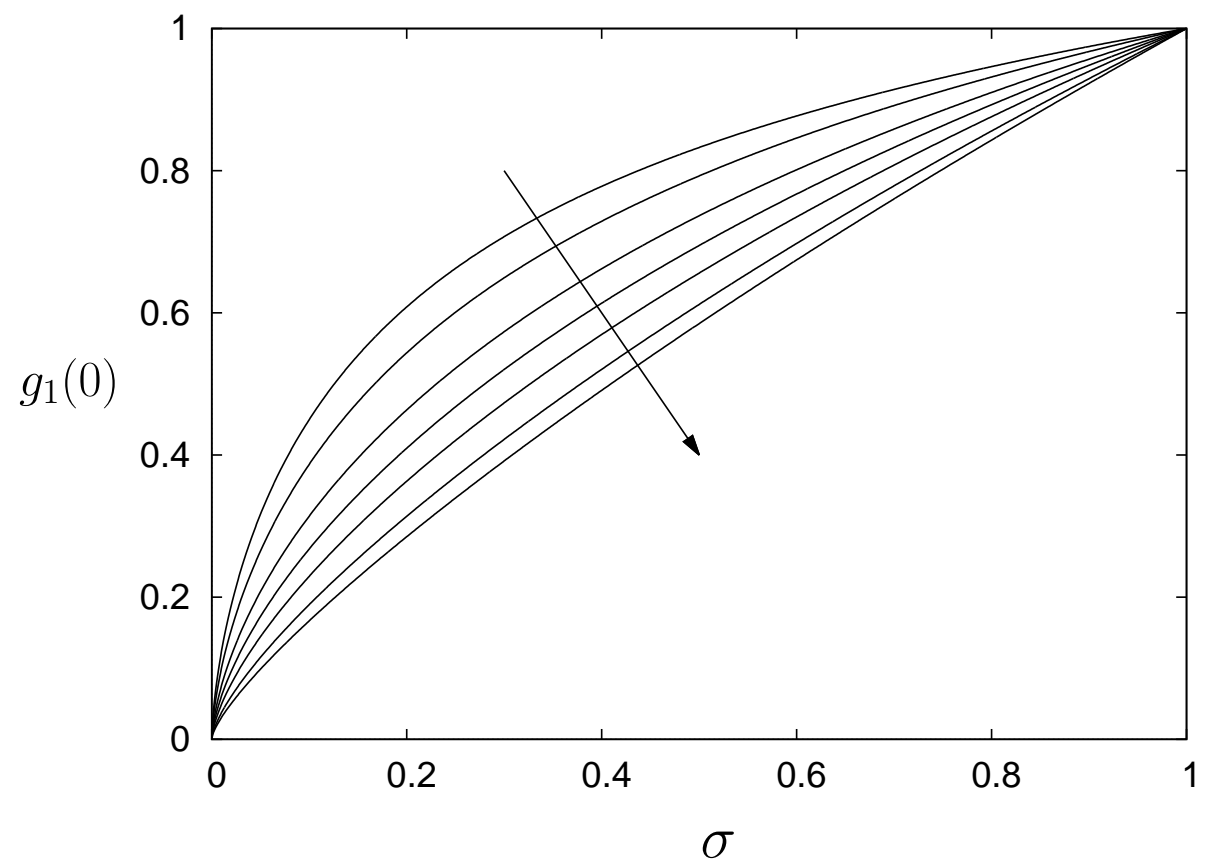

Figure 5. Upper layer interfacial azimuthal velocity $g_{1}(0)$ for positive values of $\sigma$ at $\mu=\{0.1,0.2,0.5,1.0,2.0,5.0,10.0\}$ with the arrow in the direction of increasing values of $\mu$. 


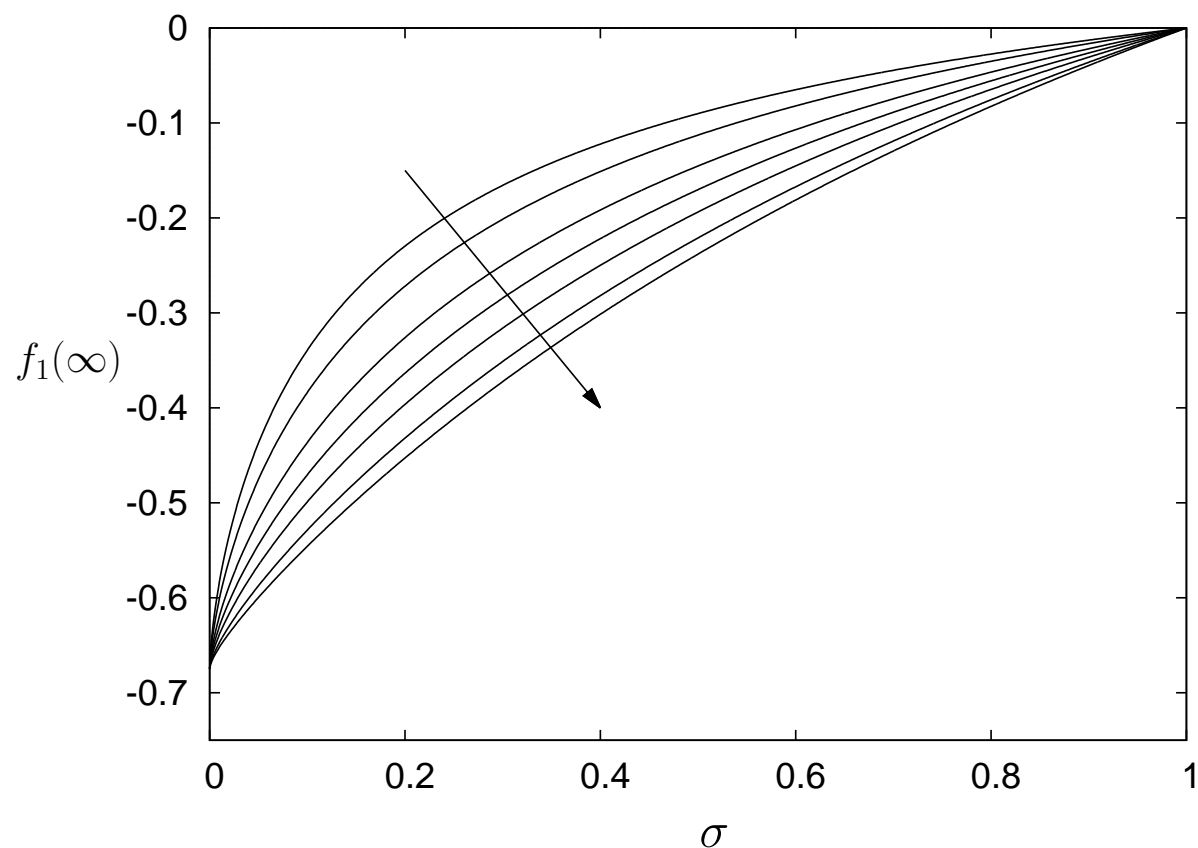

Figure 6. Variation of far-field behavior of the axial velocity parameter in the upper layer $f_{1}(\infty)$ with positive values of $\sigma$ at $\mu=\{0.1,0.2,0.5,1.0,2.0,5.0,10.0\}$ with the arrow in the direction of increasing values of $\mu$.

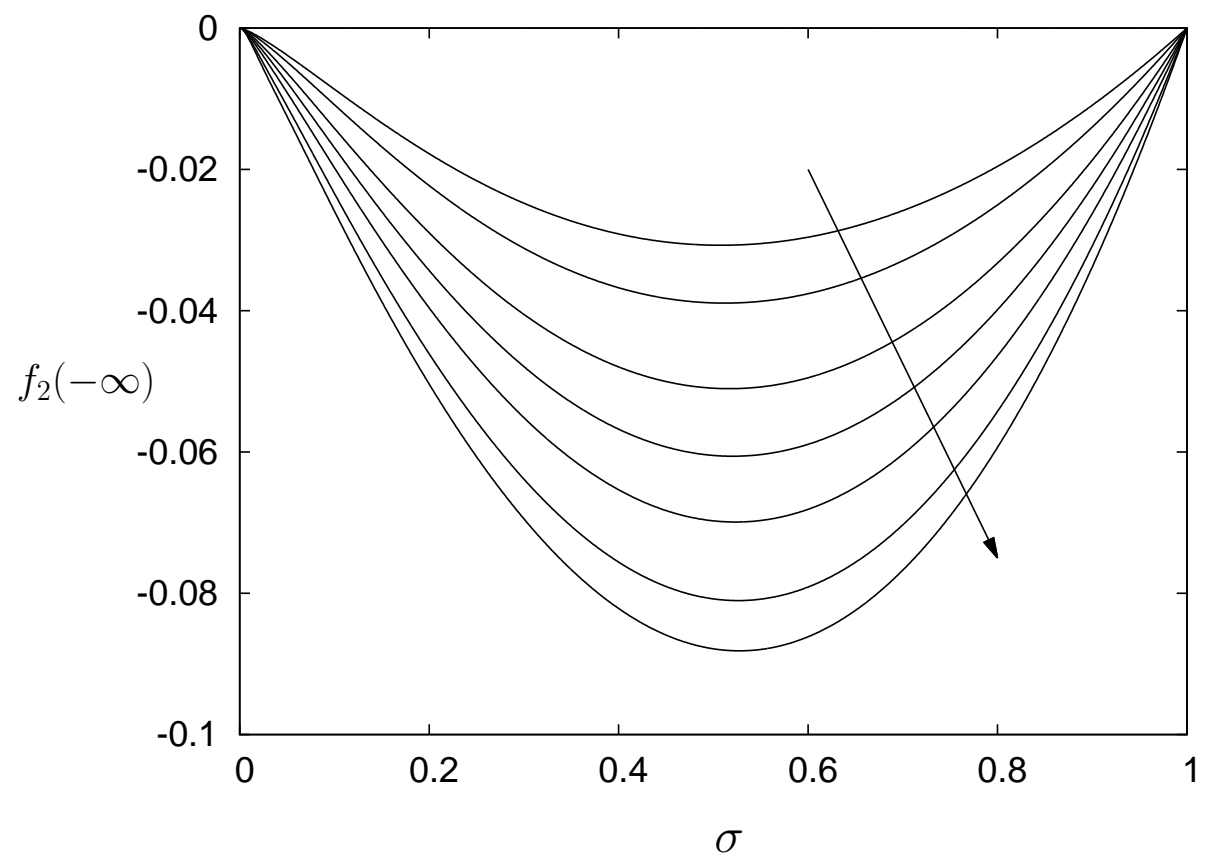

Figure 7. Variation of far-field behavior of axial velocity parameter in the lower layer $f_{2}(-\infty)$ with positive values of $\sigma$ at $\mu=\{0.1,0.2,0.5,1.0,2.0,5.0,10.0\}$ with the arrow in the direction of increasing values of $\mu$. 


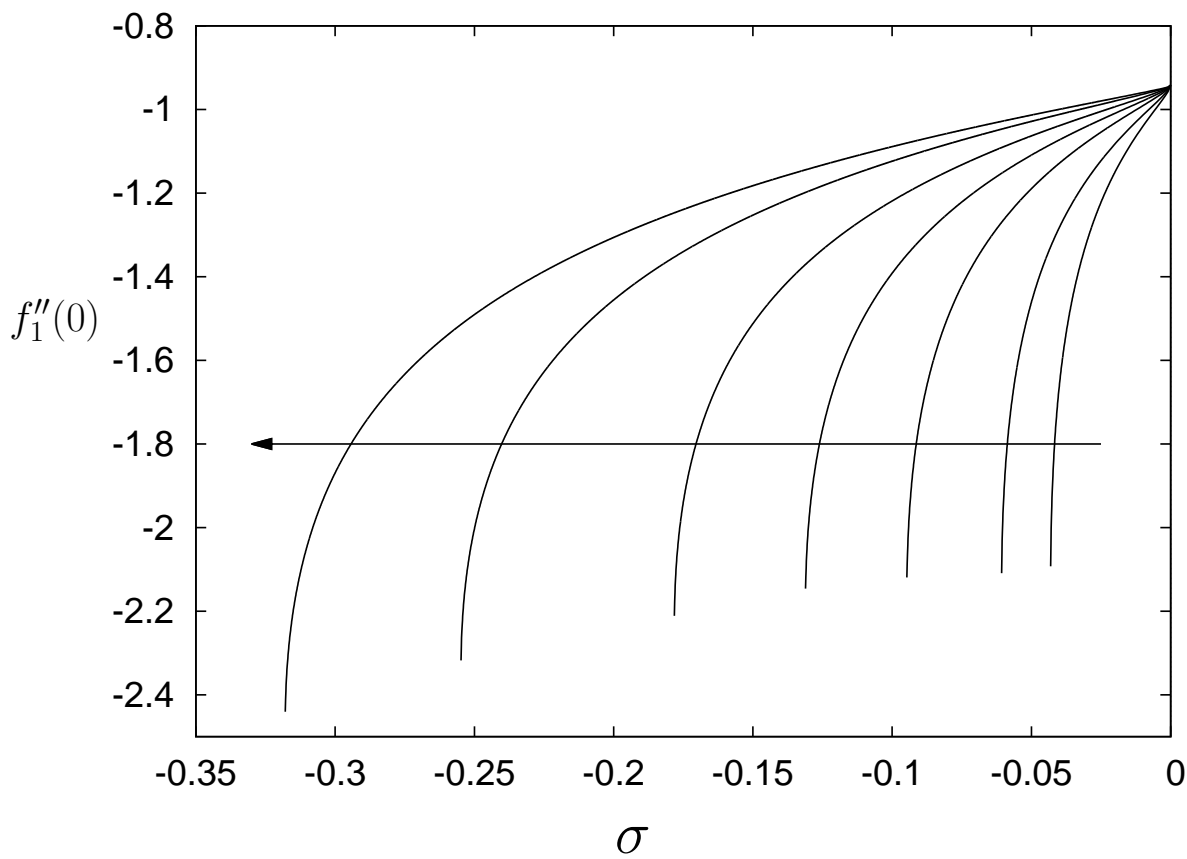

Figure 8. Variation of upper interfacial radial stress parameter $f_{1}^{\prime \prime}(0)$ with negative values of $\sigma$ at $\mu=\{0.1,0.2,0.5,1.0,2.0,5.0,10.0\}$ with the arrow in the direction of increasing values of $\mu$.

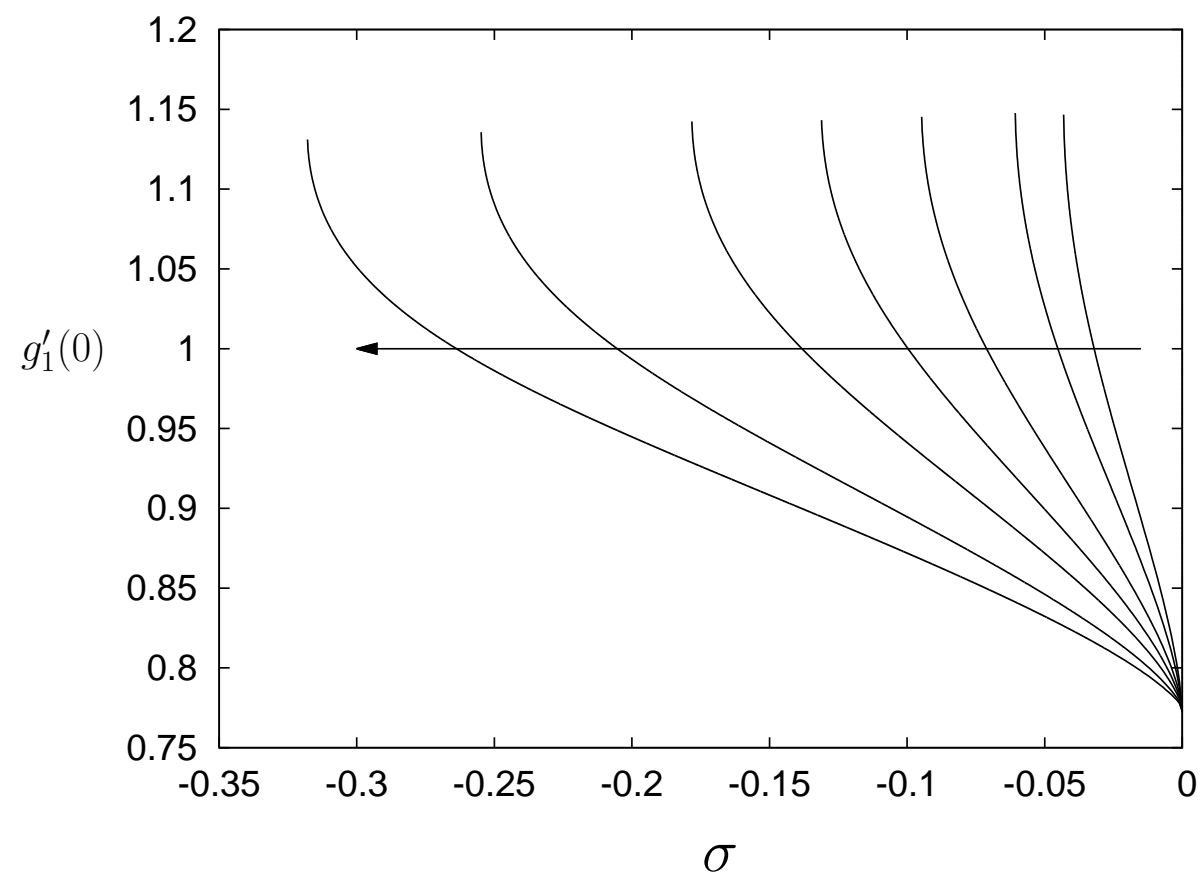

Figure 9. Variation of upper interfacial azimuthal stress parameter $g_{1}^{\prime}(0)$ with negative values of $\sigma$ at $\mu=\{0.1,0.2,0.5,1.0,2.0,5.0,10.0\}$ with the arrow in the direction of increasing values of $\mu$. 


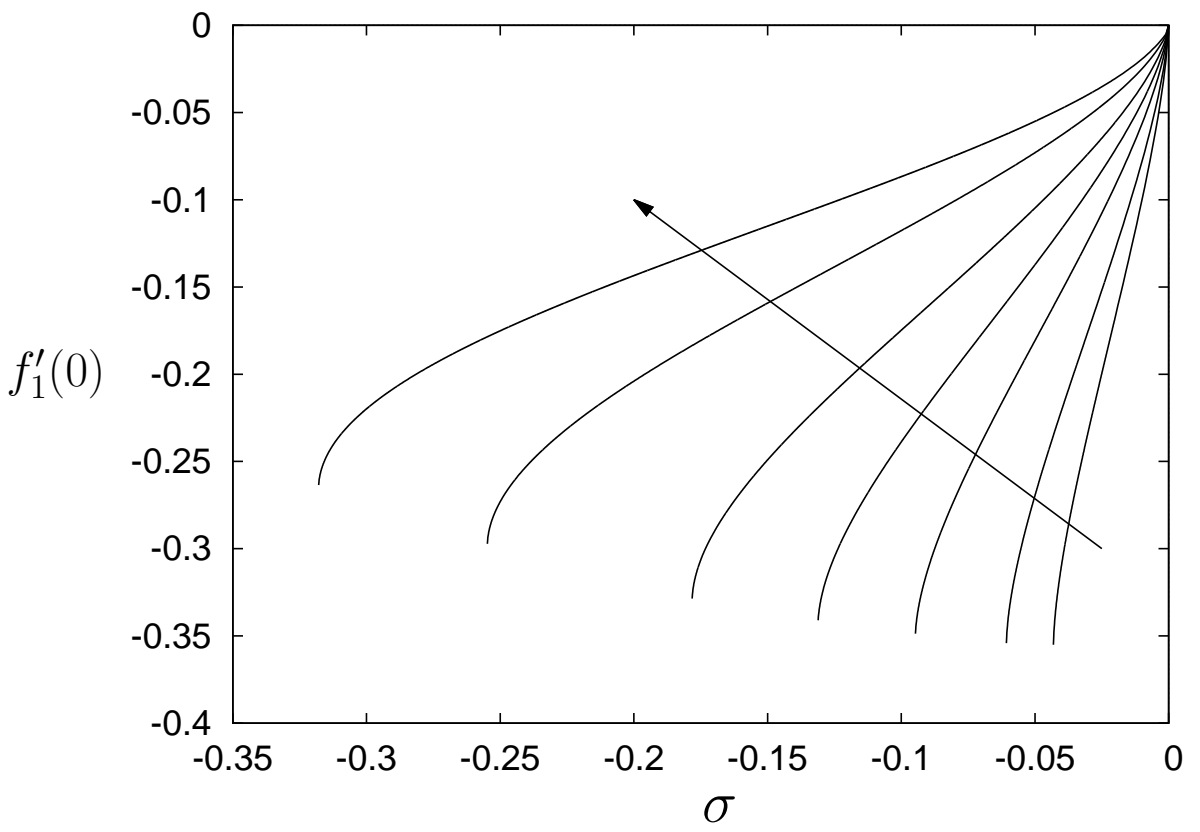

Figure 10. Upper layer interfacial radial velocity $f_{1}^{\prime}(0)$ for negative values of $\sigma$ at $\mu=\{0.1,0.2,0.5,1.0,2.0,5.0,10.0\}$ with the arrow in the direction of increasing values of $\mu$.

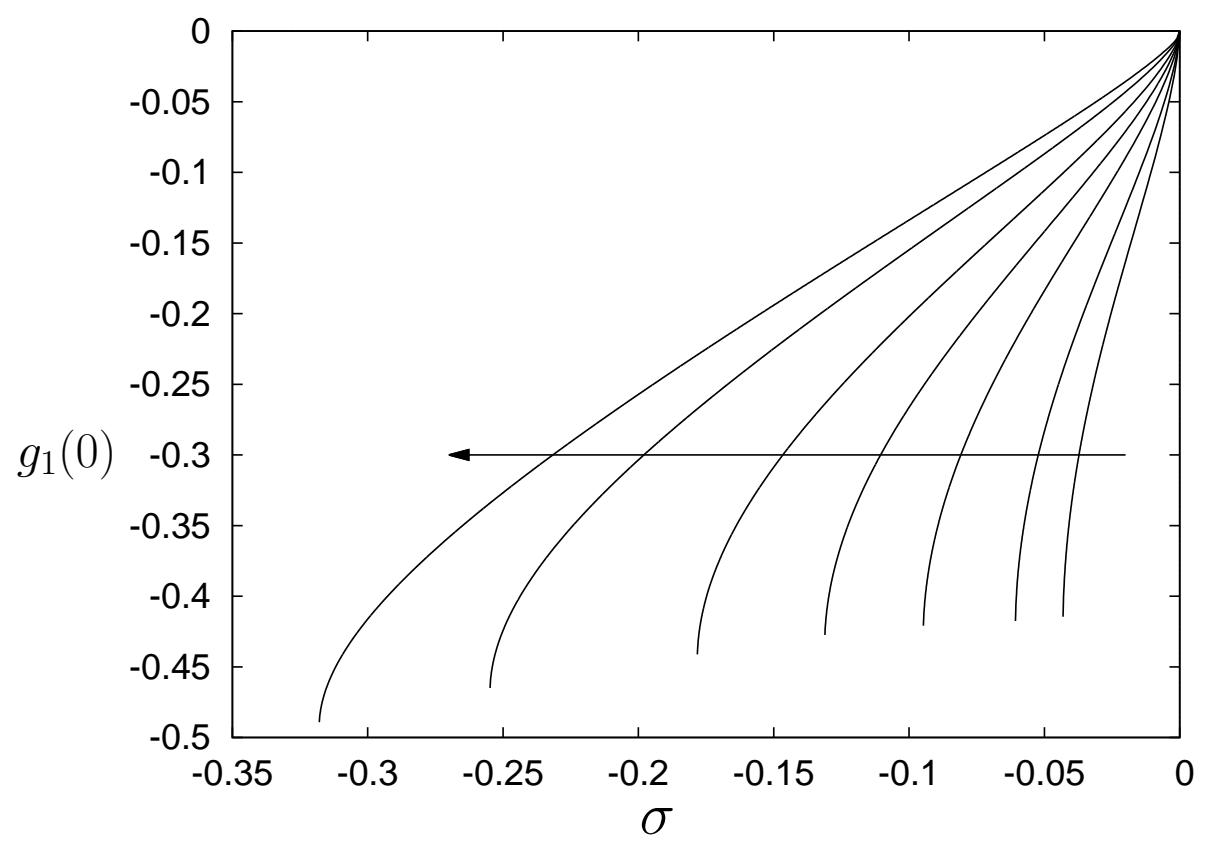

Figure 11. Upper layer interfacial azimuthal velocity $g_{1}(0)$ for negative values of $\sigma$ at $\mu=\{0.1,0.2,0.5,1.0,2.0,5.0,10.0\}$ with the arrow in the direction of increasing values of $\mu$. 


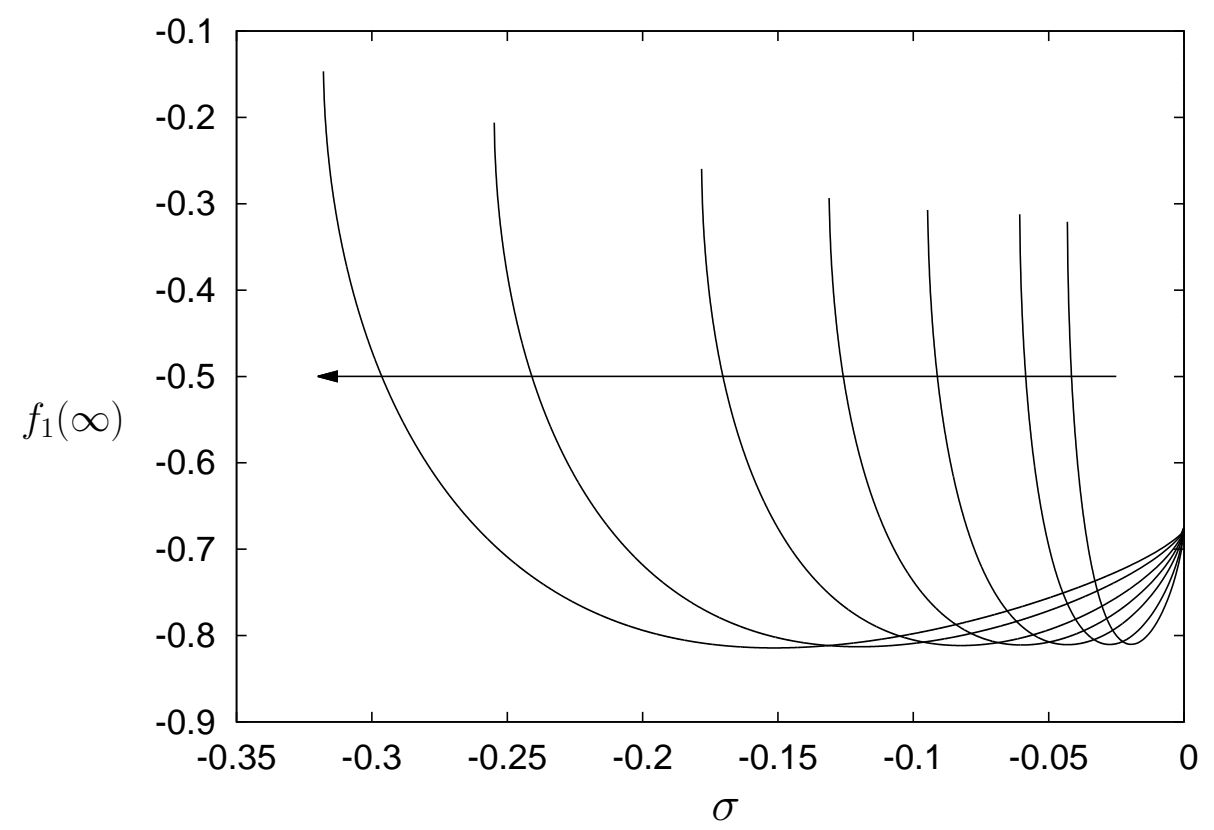

Figure 12. Variation of far-field behavior of the axial velocity parameter in the upper layer $f_{1}(\infty)$ with negative values of $\sigma$ at $\mu=\{0.1,0.2,0.5,1.0,2.0,5.0,10.0\}$ with the arrow in the direction of increasing values of $\mu$.

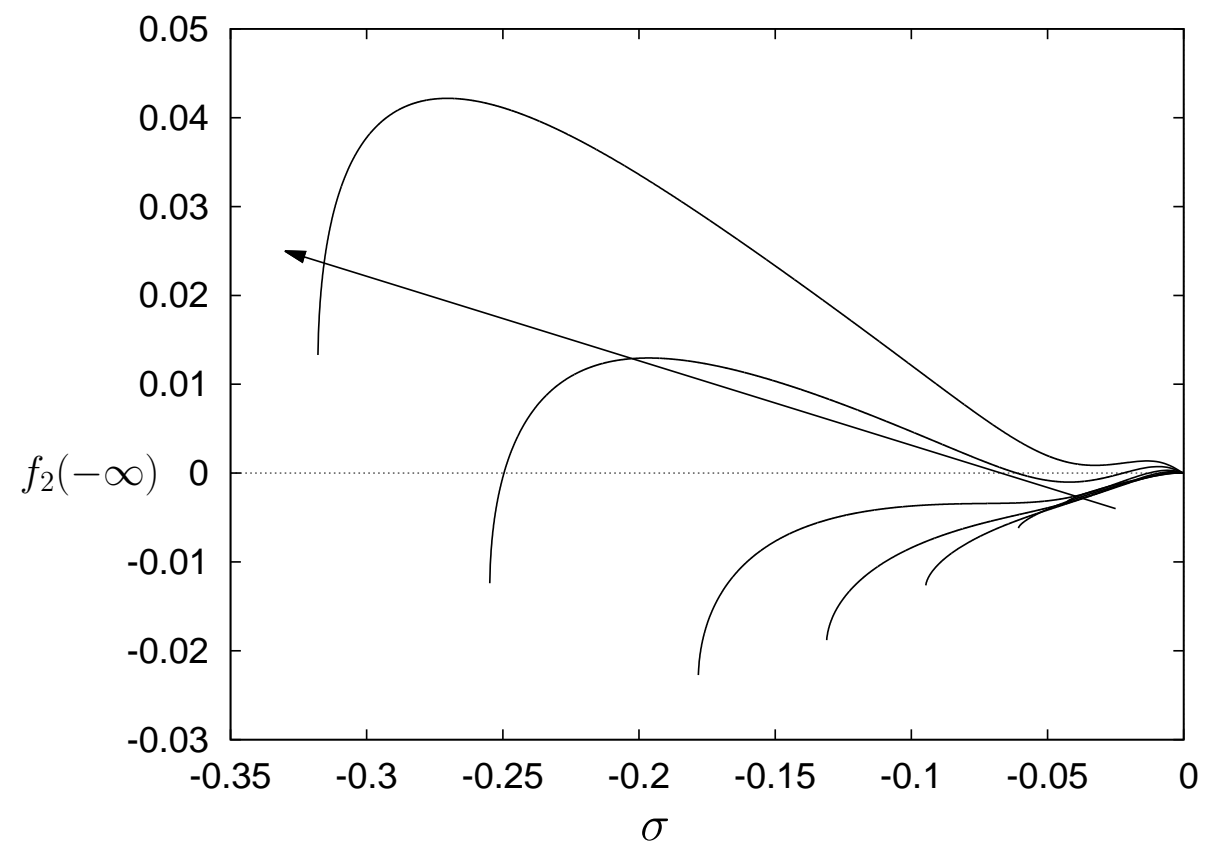

Figure 13. Variation of far-field behavior of axial velocity parameter in the lower layer $f_{2}(-\infty)$ with negative values of $\sigma$ at $\mu=\{0.1,0.2,0.5,1.0,2.0,5.0,10.0\}$ with the arrow in the direction of increasing values of $\mu$. 


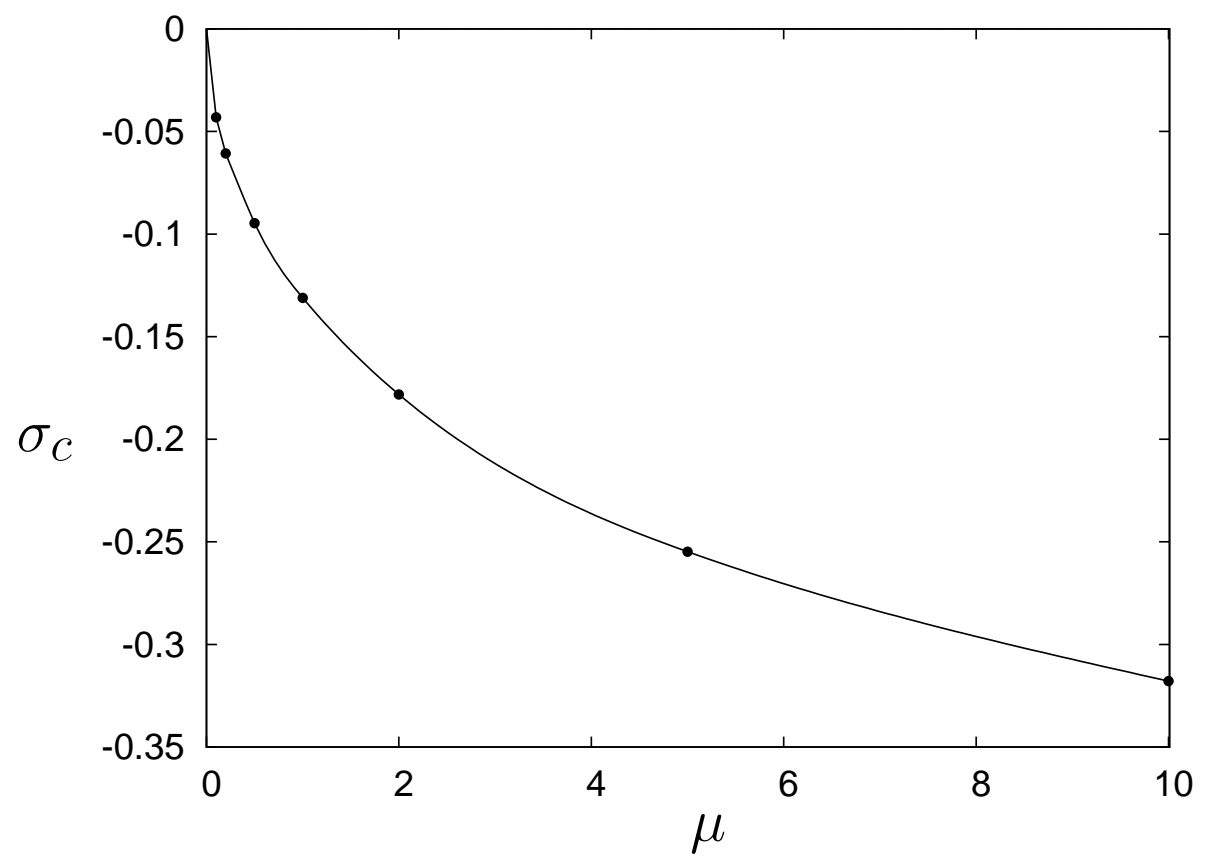

Figure 14. Variation of the limiting values $\sigma_{c}$ for negative values of $\sigma$ as a function of $\mu$.

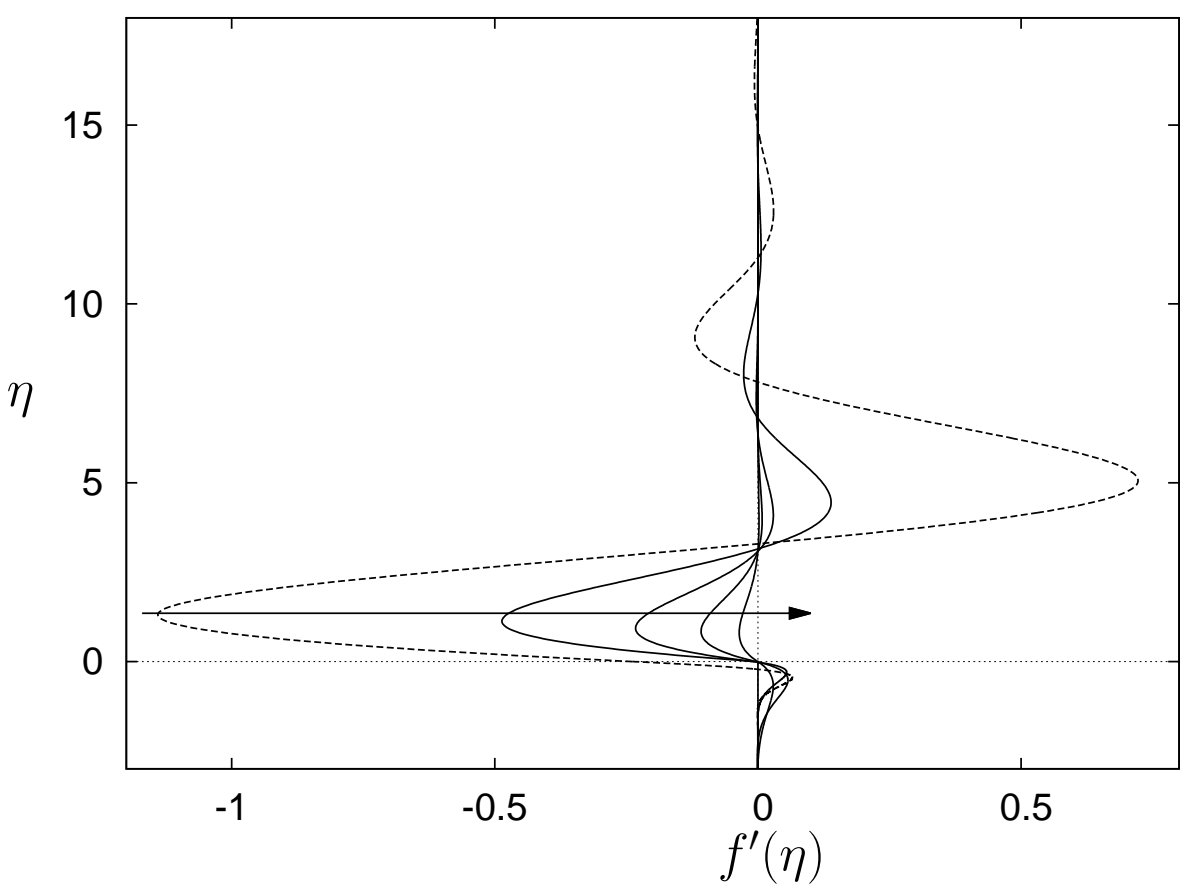

Figure 15. Plot of both upper and lower layer radial velocity profiles $f^{\prime}(\eta)$ for $\mu=1$ at $\sigma=\{-0.1,0.0,0.2,0.5,0.8\}$ with the $\sigma=-0.1$ profile plotted as the dashed line. The arrow denotes the direction of increasing $\sigma$. 


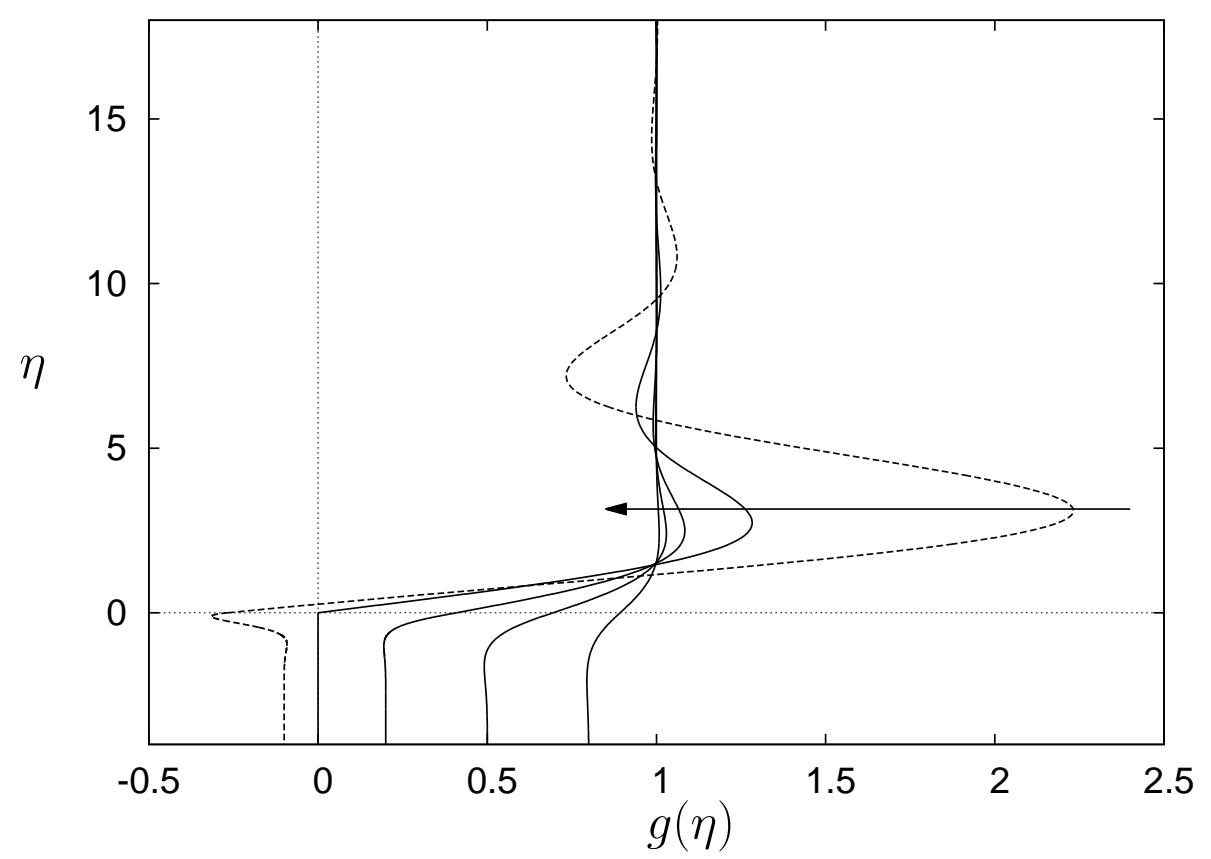

Figure 16. Plot of both upper and lower layer azimuthal velocity profiles $g(\eta)$ for $\mu=1$ at $\sigma=\{-0.1,0.0,0.2,0.5,0.8\}$ with the $\sigma=-0.1$ profile plotted as the dashed line. The arrow denotes the direction of increasing $\sigma$.

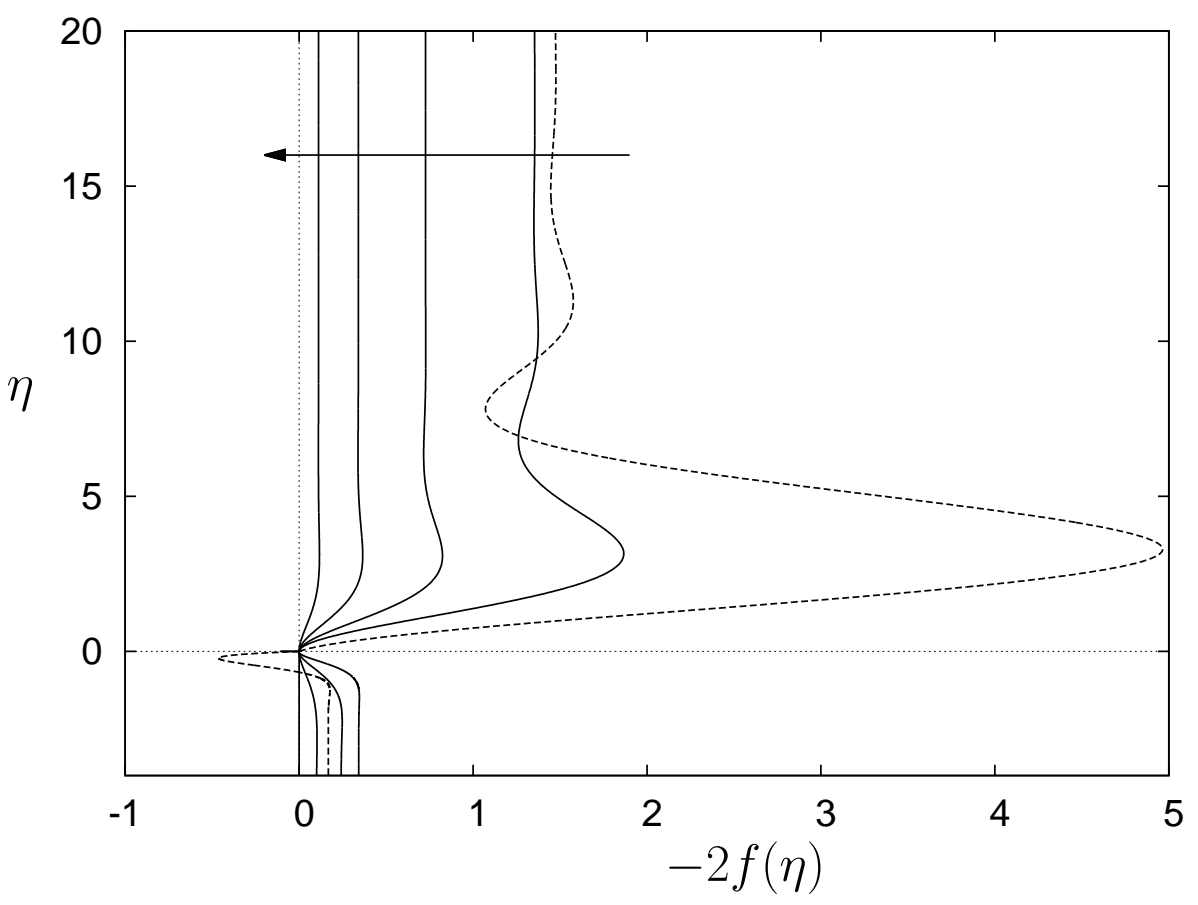

Figure 17. Plot of both upper and lower layer axial velocity profiles $-2 f(\eta)$ for $\mu=1$ at $\sigma=\{-0.1,0.0,0.2,0.5,0.8\}$ with the $\sigma=-0.1$ profile plotted as the dashed line. The arrow denotes the direction of increasing $\sigma$. 